\title{
Method of temperature compensation to spin-valve sensor based on PGA
}

\author{
Xiaofeng Shen ${ }^{1, \text { a }}$, Huachen Zhu ${ }^{1, b}$, Ru Bai ${ }^{* 1, c}$, Zhenghong Qian*1,d \\ ${ }^{1}$ Center for Integrated Spintronic Devices, Hangzhou Dianzi University,Hangzhou 310018, China. \\ asshenxiaofeng91@163.com, bhuachen_zhu@126.com, 'bairu@hdu.edu.cn, 'zqian@hdu.edu.cn.
}

Keywords: Spin-valve sensor; Temperature compensation; Programmable differential amplifier Abstract. The spin-valve sensor has been widely used in automotive electronic systems, industrial control systems, medical equipment and other technical fields, due to its advantages of high sensitivity, low energy consumption, as well as small size. The sensitivity of the spin valve sensor is affected by temperature, especially in the environment with a large temperature variation. Therefore, it is necessary to carry out a temperature compensation to the spin-valve sensor for high precision detection applications. In this paper, the temperature characterization of the spin-valve sensor, which is fabricated by our research group, was conducted. And the output signal of sensor was adjusted to compensate the temperature drift by using a programmable differential amplifier, LMH6881 of TI Company. Compared with the algorithms of software compensation, our temperature compensation method by using the amplifier has the advantages of easy operation, high efficiency and high precision.

\section{Introduction}

In 1988, two scientists, Peter Grünberg and Albert Fert, independently discovered a noval physical phenomena -- Giant Magneto-Resistance effect (GMR), and shared the Nobel Prize in Physics in 2007 [1]. Then, GMR sensors were developed, including the spin-valve sensors, the multilayer film sensors [2]. Compared with Hall sensors and AMR sensors, spin-valve sensors have many advantages of small size, high sensitivity, low consumption and good stability, and have been widely used in industrial control, automotive electronics, medical electronics, smart grid, intelligent transportation and other fields [3].

Spin-valve sensor usually has a wheatstone bridge structure, composed of four giant magnetic resistance sensing elements [4]. It shows good thermal stability, and remains consistent in a small range of temperature variation. However, when applied in a large range of temperature variation, a temperature compensation is needed to calibrate the output of the spin-valve sensors, in order to complete high precision measurement, such as smart meters, security systems. A very common method of temperature compensation is software-based compensation method [5], which establishes mathematical model for the output data of sensors, and analyzes and processes through a dedicated algorithm, ultimately realizes temperature compensation, such as least square method [6], particle swarm optimal algorithm [7], BP neural network algorithm [8]. But these methods have complicated calculation steps and need a long time optimization.

This paper introduces a temperature compensation method based on programmable differential amplifier for the spin-valve sensor with a magnetic shielding structure, and designs a circuit system which can adjust the output of sensor, and achieve temperature drift compensation effectively. The method has the advantages of simple process, high efficiency, and excellent real-time performance.

\section{System circuit design}

The hardware configuration of this temperature compensation system for spin-valve sensor is shown in Fig. 1. It includes four parts: PGA, MCU, temperature acquisition module, as well as LCD display module. The system's working principle is that the MCU receives the temperature and determines, 
then controls the selection of the corresponding gain of PGA to adjust the output of the sensor, and shows the parameters on LCD.

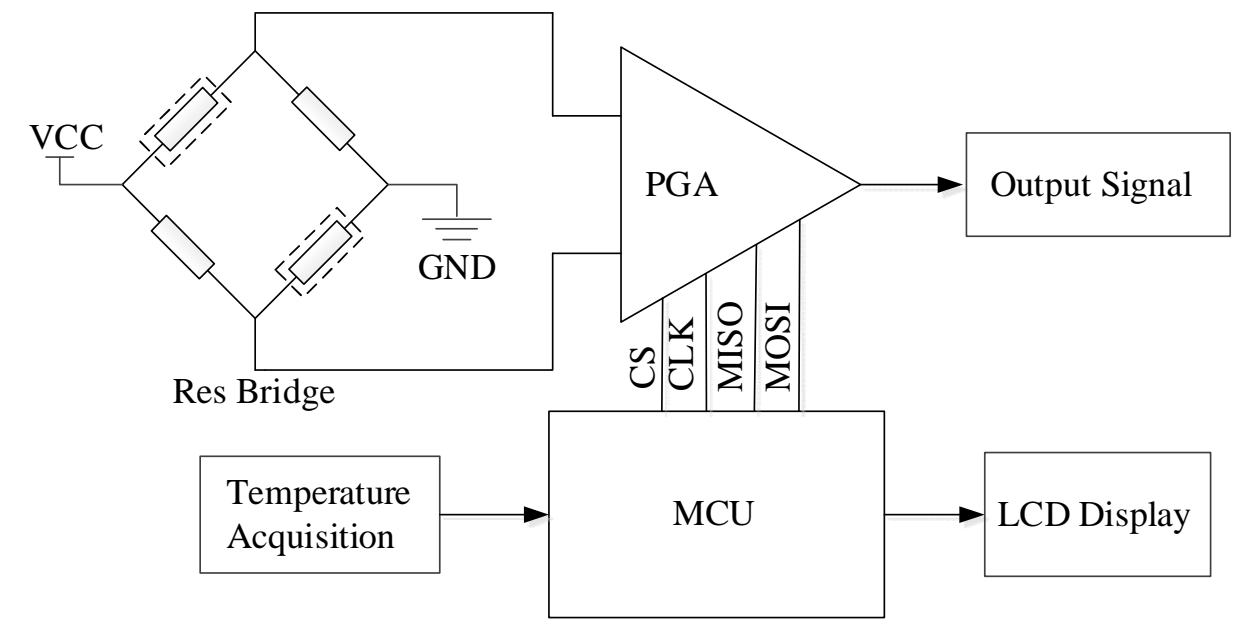

Fig. 1. System hardware structure diagram

\section{Spin-valve sensor}

In this paper, the spin-valve sensor is designed by our group, Center for Integrated Spintronic Devices in Hangzhou Dianzi University [9]. It has a wheatstone bridge structure as shown in Fig. 2. The wheatstone bridge is composed by four giant magnetic resistors. Two resistors (R1, R3) are covered by NiFe soft magnetic shielding layer, while two other resistors (R2, R4) are not covered and able to response to external magnetic field. When applied in an external magnetic field, R1 and R3 are not affected, R2 and R4 change with the external magnetic field. Therefore, the output of the bridge is changed accordingly. The output of the whole bridge reflects the strength of the external magnetic field. In this structure, NiFe soft magnetic shielding layer can not only shield the influence of the magnetic field to R1, R3, but also can strengthen the magnetic field near R2, R4 [10].

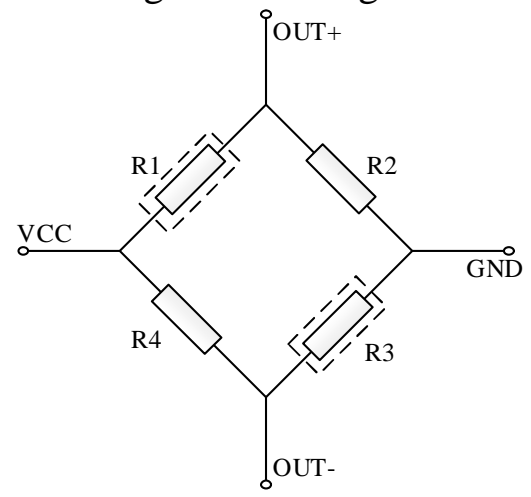

Fig. 2. Wheatstone bridge structure of the spin-valve sensor

\section{Signal processing module}

The signal processing module is designed by using LMH6881, which is a high speed and high performance amplifier of TI Company. It has the ability to input double-end differential signal, and the input impedance is $100 \Omega$. It also supports $50 \Omega$ single-ended signal source and application of DC and AC coupling. LMH6881 has $2.4 \mathrm{GHz}$ bandwidth and high linearity of $44 \mathrm{dBm}$ OIP3, and provides excellent noise and distortion resistance, which is suitable for all kinds of signals.

LMH6881 will support two modes of control for its gain: a parallel mode and a serial mode. In parallel mode the gain can be changed in 2-dB steps with a 4-bit gain control bus. The serial interface allows a great deal of flexibility in gain programming and reduced board complexity. Its gain step is $0.25 \mathrm{~dB}$, and it communicates with control circuit to realize automatic gain-control through the serial peripheral interface (SPI) bus.

This paper places the LMH6881 into serial mode, Table 1 shows the data format of register. Eq. 1 can get the control command word (CMD). 
Gain $[\mathrm{dB}]=26-$ Register*0.25

Table 1. The gain control register in serial mode of LMH6881 [unit:dB]

\begin{tabular}{lllllllll}
\hline Bit & 7 & 6 & 5 & 4 & 3 & 2 & 1 & 0 \\
\hline Configuration & Reserved & 16 & 8 & 4 & 2 & 1 & 0.5 & 0.25 \\
\hline
\end{tabular}

Temperature acquisition module

DS18B20 is used in temperature acquisition module, which works with 1-wire bus protocol to transmit data without external components. As is shown in Fig. 3, DQ is data I/O. It can measure temperatures from $-55^{\circ} \mathrm{C}$ to $+125^{\circ} \mathrm{C}$, meet the requirements of the sensor operating temperature range.

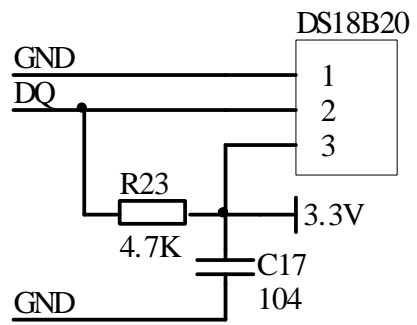

Fig. 3 DS18B20 schematic diagram

\section{MCU and LCD display module}

STM32F103C8T6 as microcontroller is a high performance, low cost, low power consumption of the Cortex-M3 CPU, its highest working frequency is $72 \mathrm{MHz}$ and the working voltage is $3.3 \mathrm{~V}$. It integrates internal SPI, the system sets the STM32 in master mode, and LMH6881 in slave mode, they communication with full-duplex mode. LCD display module for this experiment shows the initial information of the hardware, and displays the temperature and the amplifier gain in a real-time.

\section{System software design}

Fig. 4. shows program flow diagram. The table-lookingup methods is adopted as software algorithm, which get the corresponding CMD to control the gain of the amplifier dynamically in different temperatures, and set a threshold to reduce the gain jumping, then make the measurement process stable.

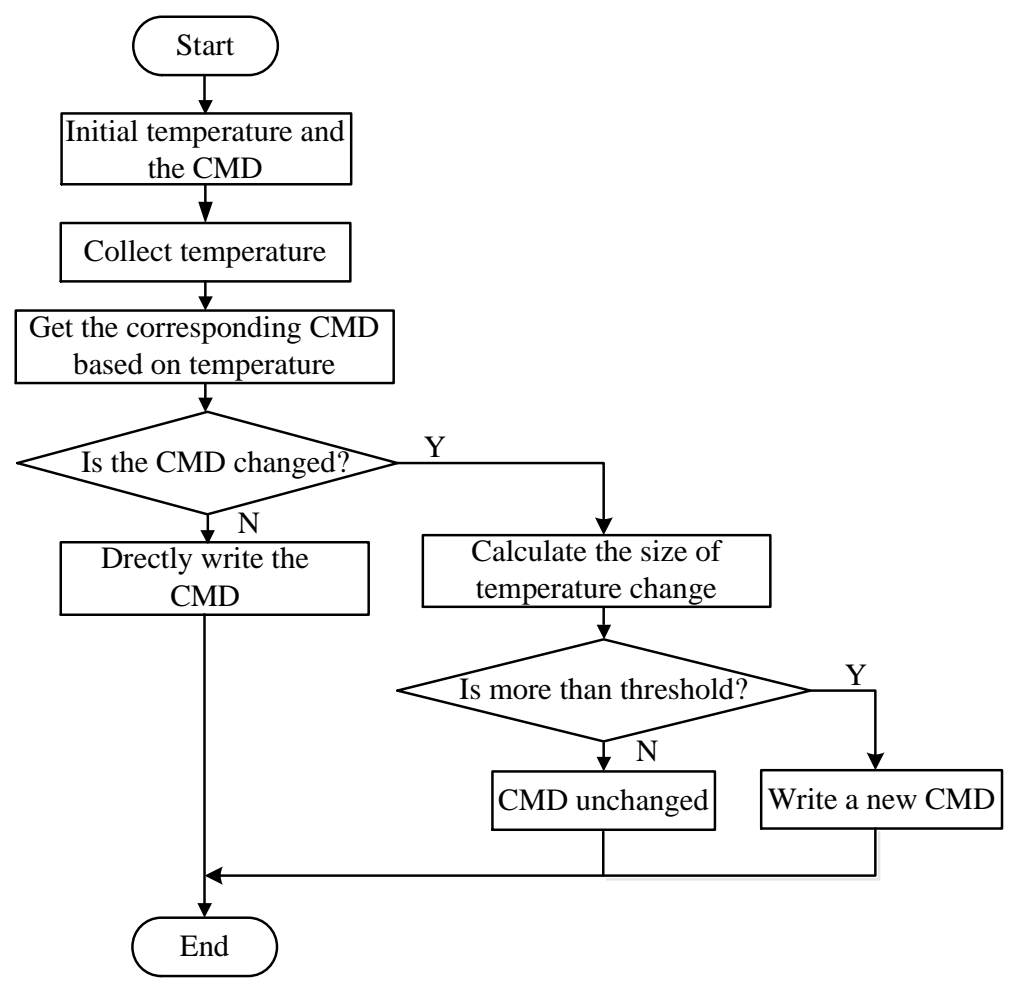

Fig. 4. Program flow diagram 
Table 2 demonstrates the optimal value of the table-lookingup methods, including temperature range, multiple and corresponding CMD.

Table 2. Corresponding amplifier gain and CMD in different temperatures [unit: ${ }^{\circ} \mathrm{C}$ ]

\begin{tabular}{lccccccccccc}
\hline $\begin{array}{l}\text { Temperature } \\
(>=)\end{array}$ & -25.0 & -13.5 & -1.0 & 11.1 & 22.8 & 34.2 & 45.3 & 56.1 & 66.6 & 76.7 & 85 \\
\hline Multiple & 2.175 & 2.239 & 2.304 & 2.304 & 2.371 & 2.441 & 2.512 & 2.585 & 2.661 & 2.738 & 2.818 \\
CMD & 0X4C & 0X4B & 0X4A & 0X4A & 0X49 & 0X48 & 0X47 & 0X46 & 0X45 & 0X44 & 0X43 \\
\hline
\end{tabular}

This design uses the table-lookingup methods to get automatic gain-control conveniently, but in practice, the temperature gathered by DS18B20 will jump up and down to a certain extent, the small scale changing in overlap part of temperature area leads to CMD changes frequently, it greatly reduce the efficiency and stability. Therefore, the algorithm needs to be optimized, the specific plan is as following: Set a threshold of temperature $0.5^{\circ} \mathrm{C}$ and initialize the temperature and CMD to be a extreme value, to ensure that the first temperature difference exceeds the threshold, thus look up table accurately. When the one decimal places of the temperature jump up and down at the overlap region, the gain will change synchronously. So when the CMD changes, and the temperature difference does not exceed the threshold, then write a new CMD, or keep the CMD unchanged.

\section{Experiment and result analysis}

Fig. 5 presents high-low temperature testing platform for magnetic sensor. The platform is mainly composed of three-axis Helmholtz coil and high-low temperature test box. The three-axis Helmholtz coil is drived by three programmable current source. It can scan magnetic field respectively in the X, $\mathrm{Y}, \mathrm{Z}$ three directions, and desktop multimeter can test magnetic sensor cooperate with it. The high and low temperature test box can work at $-60 \sim 150^{\circ} \mathrm{C}$.

In this paper, three-axis Helmholtz coil scan magnetic field between $-6 \sim 6$ Oe along the spin-valve sensor sensitive-axis, step of magnetic field scanning is 0.060 e. The range of experimental temperature is $-25 \sim 85^{\circ} \mathrm{C}$, and test interval is $10^{\circ} \mathrm{C}$, covers temperature range of consumer electronics application.

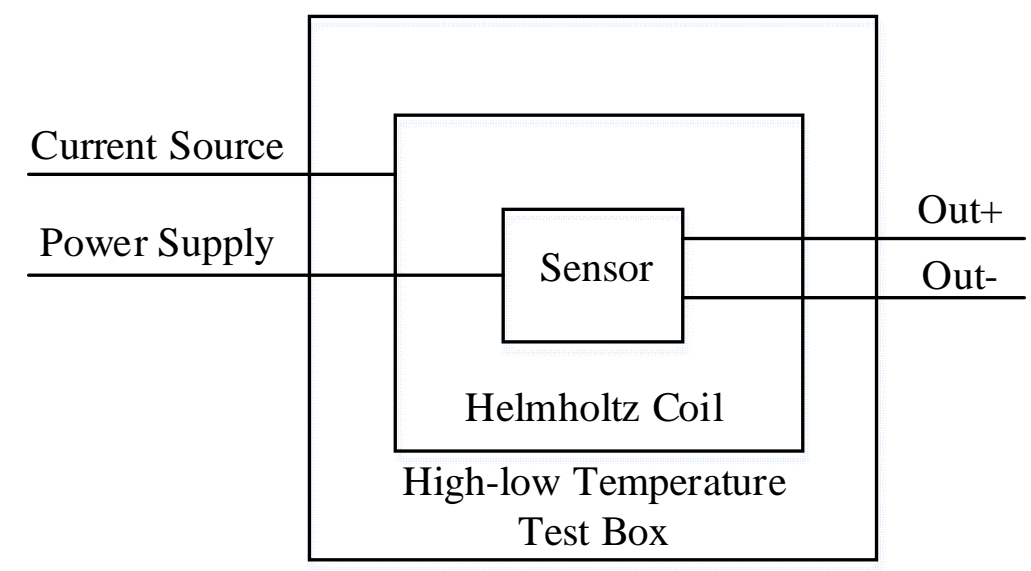

Fig. 5. Temperature testing platform

\section{Temperature characteristic experiment}

Based on project preparation and testing, the sensitivity of the sensor chip in $-25 \sim 85^{\circ} \mathrm{C}$ is demonstrated with dot in Fig. 6, the sensitivity is in a linear relationship with temperature, and establish the mathematical model by a linear fitting method, as is shown in the Eq. 2.

$\mathrm{S}(\mathrm{T})=\left(1-\mathrm{C}_{\mathrm{T}} *(\mathrm{~T}-25)\right) * \mathrm{~S}_{0}$

Among this formula, $\left(1-\mathrm{C}_{\mathrm{T}} *(\mathrm{~T}-25)\right)$ is the temperature coefficient of sensor's sensitivity, and the $\mathrm{C}_{\mathrm{T}}$ is calculated to be 0.0025 by a large number of experimental data fitting. 


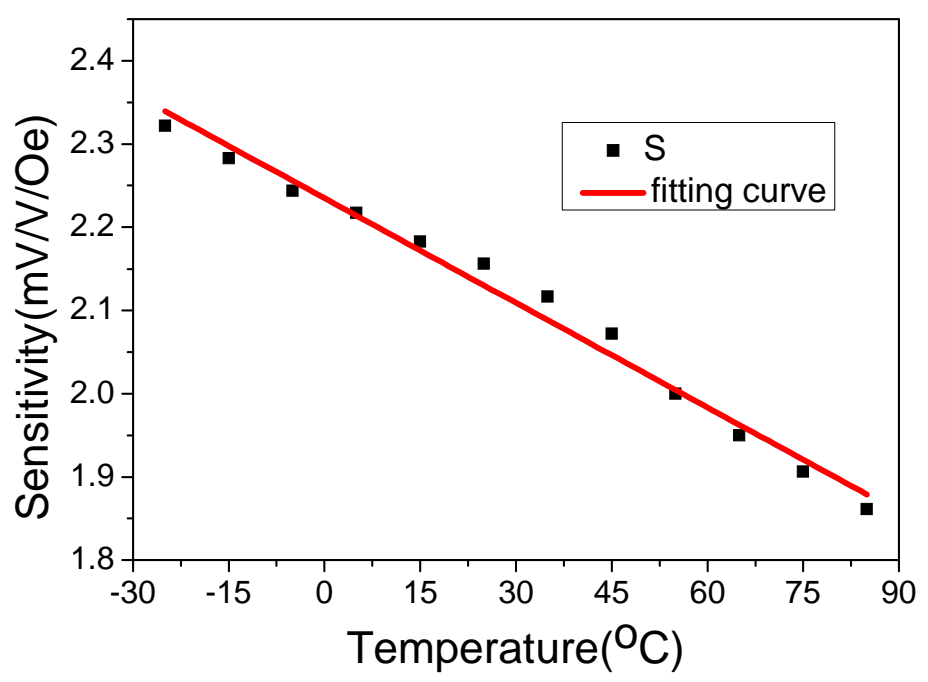

Fig. 6. Sensor sensitivity vs different temperature

\section{Effect of temperature compensation}

Sensitivities of the sensor get the corresponding adjustment in different temperatures through the temperature compensation system. After the experiment for many times, the average of sensitivities which obtained in different temperatures are calculated. Table 3 shows the difference $\left(\Delta \mathrm{S}_{0}\right)$ between original sensitivity $\left(\mathrm{S}_{0}\right)$ and the sensitivity in calibration temperature $\left(25^{\circ} \mathrm{C}\right)$, and the difference $(\Delta \mathrm{S})$ between adjusted sensitivity $(\mathrm{S})$ and the adjusted sensitivity in calibration temperature $\left(25^{\circ} \mathrm{C}\right)$.

Table 3: Comparision of sensitivities before and after temperarure compensation

\begin{tabular}{lllll}
\hline Temperature $\left({ }^{\circ} \mathrm{C}\right)$ & $\begin{array}{l}\text { original } \\
\text { sensitivity }\left(\mathrm{S}_{0}\right)\end{array}$ & $\begin{array}{l}\text { adjusted } \\
\text { sensitivity }(\mathrm{S})\end{array}$ & $\triangle \mathrm{S}_{0}$ & $\triangle \mathrm{S}$ \\
\hline-25 & 2.333 & 2.121 & 0.150 & -0.049 \\
-15 & 2.317 & 2.099 & 0.134 & -0.071 \\
-5 & 2.283 & 2.128 & 0.100 & -0.042 \\
5 & 2.250 & 2.163 & 0.067 & -0.007 \\
15 & 2.217 & 2.142 & 0.034 & -0.028 \\
25 & 2.183 & 2.170 & 0.000 & 0.000 \\
35 & 2.133 & 2.121 & -0.050 & -0.049 \\
45 & 2.067 & 2.142 & -0.116 & -0.028 \\
55 & 2.033 & 2.149 & -0.150 & -0.021 \\
65 & 1.983 & 2.142 & -0.200 & -0.028 \\
75 & 1.917 & 2.128 & -0.266 & -0.042 \\
85 & 1.867 & 2.135 & -0.316 & -0.035 \\
\hline
\end{tabular}

The data in Table 3 shows that the maximum absolute value $\Delta \mathrm{S}_{0}$ is $0.316 \mathrm{mV} / \mathrm{V} / \mathrm{Oe}$, the relative error is $14.6 \%$, and the maximum absolute value $\Delta \mathrm{S}$ is $0.071 \mathrm{mV} / \mathrm{V} / \mathrm{Oe}$, the relative error is $3.2 \%$. Fig. 7 intuitively shows the sensitivities which are tested before and after the temperature compensation. Line $S_{0}$ shows that the original sensitivities change linearly with the temperature, and line $S$ under the standard line $25^{\circ} \mathrm{C}$ has small fluctuation, and close to be a straight line. Thus, temperature compensation effect is obvious. The sensitivites in different temperatures are adjusted nearly to the same. 


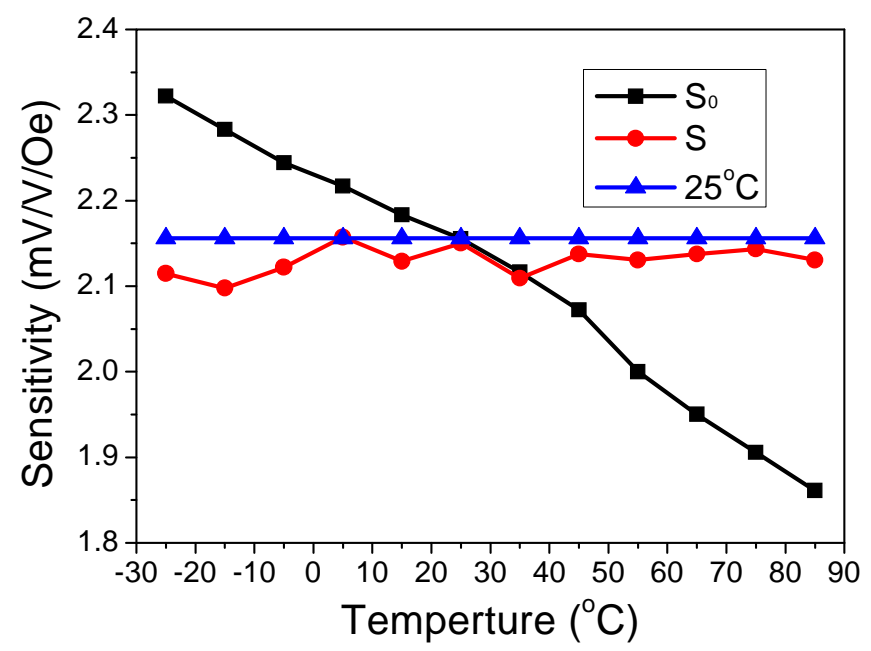

Fig. 7. Sensitivities before and after temperature compensation

\section{Conclusions}

In this paper, a set of temperature compensation scheme based on programmable gain amplier for spin-valve sensor is designed and fabricated. The working principle and temperature characteristic of the spin-valve sensor is analyzed and tested to evaluate the effect of the compensation method. The programmable differential amplifier, LMH6881could dynamically control the output gain of the spin-valve sensor, and realize the compensation to the temperature drift. The result shows that the effect is obvious. Compared with software compensation methods, our method has simple steps and strong real-time performance.

\section{Acknowledgements}

This work was financially supported by National High-tech R\&D Program of China (No. 2014AA032904).

\section{References}

[1] W.Y. Lai: Chinese Journal of Nature. Vol. 29-6(2007), p. 348-352. In Chinese.

[2] L. James and S.E. Alan: IEEE Sensors journal. Vol. 6-3(2006), p. 631-649.

[3] L.L. Hu, C. Liu and G.H. Gai: Journal of Transduction Technology. Vol. 25-4(2012), p. 468-471. In Chinese.

[4] S.N. Mao, N. Amin and E. Murdock: Journal of applied physics. Vol. 83-11(1998), p. 6807-6809.

[5] Y.P. Du and X.Y. He. Electronic Design Engineering. Vol. 17-6(2009), p. 63-64. In Chinese.

[6] J.B. Yu, J. Li, Q.L. Dai, D. Li and Y.P. Lv: IEEE Transactions on Instrumentation and Measurement. Vol. 64-1(2015), p. 204-211.

[7] X.Q. Wang and J.Q Fu: Automation Instrument. Vol. 32-10(2011), p. 69-71. In Chinese.

[8] J.L. Li, W.J. Wang, F. Jiao, J.C. Fang and T. Yu: Journal of Applied Mathematics, Vol. 2014-901539(2014), p. 1-11.

[9] H.C. Zhu, Z.H. Qian, L. Hu and R. Bai: Materials protection. Vol. 46-2(2013), p. 164-166. In Chinese. 
[10] Z.H. Qian, R. Bai, C.K. Huang and J.D. Wu: Instrument Technique and Sensor. Vol. supplement(2009), p. 96-101. In Chinese. 